SMUHEP/02-16

\title{
Indispensability of Residual Gauge Fields and Extended Hamiltonian Formalism in Light-Front Quantization of Gauge Fields
}

\author{
Y. Nakawaki ${ }^{\mathrm{a}}$ and G. McCartor ${ }^{\mathrm{b}}$

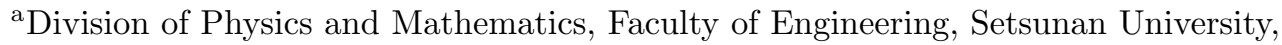 \\ Osaka 572-8508, Japan \\ ${ }^{\mathrm{b}}$ Department of Physics, Southern Methodist University, \\ Dallas TX 75275, USA
}

We consider introducing residual gauge degrees of freedom into the conventional formulation of the light-front quantization of gauge field theories. For that purpose we construct the canonical formulation of axial gauge fields of the type $n^{\mu} A_{\mu}=0$ in auxiliary coordinates: $x^{\mu}=\left(x^{\tau}, x^{\sigma}, x^{1}, x^{2}\right)$, where $x^{\tau}=x^{0} \sin \phi+x^{3} \cos \phi$ and $x^{\sigma}=x^{0} \cos \phi-x^{3} \sin \phi$. We then show that, irrespective of the quantization surface, and irrespective of the gauge fixing condition, residual gauge fields are indispensable in implementing the Mandelstam-Leibbrandt prescription and in regularizing the infrared divergences which are inherent in the canonical quantization of space-like axial gauge fields. With the residual gauge fields in place, we find that the infrared divergences are regulated with the Mandelstam-Leibbrandt prescription in the light-front formulation obtained as the limit $\phi \rightarrow \pi / 4$ of the $n^{2}=0$ case. In addition we show that, because an explicit quantization surface dependence does not appear in the $n^{2}=0$ case, the light-front temporal gauge limit $\phi \rightarrow \pi / 4-0$ agrees with the light-front spatial gauge limit $\phi \rightarrow \pi / 4+0$ and that the perturbative Hamiltonian in the light-front formulation consists of physical degrees of freedom integrated over the hyperplane $x_{l}^{+}=\left(x^{0}+x^{3}\right) / \sqrt{2}=$ constant and residual degrees of freedom integrated over the hyperplane $x_{l}^{-}=\left(x^{0}-x^{3}\right) / \sqrt{2}=$ constant.

\section{Introduction}

In spite of extensive studies of the light-front quantization of gauge fields, introducing residual gauge degrees of freedom into the theories has remained as an open issue. One knows that they have to be introduced somehow, because lightfront quantization makes use of space-like axial gauges, so that one cannot construct canonical formulations by making use of only physical degrees of freedom without encoutering infrared difficulties [1] and because equivalence between the formulation in ordinary coordinates [2] and the light-front formulation requires their introduction, at least in perturbation theory.

A first step in this direction has been taken recently by Morara and Soldati [3]. They have constructed the canonical light-front temporal gauge formulation of QED, where $x_{l}^{+}=\left(x^{0}+x^{3}\right) / \sqrt{2}$ is chosen as the evolution parameter and $A_{+}^{(l)}=$ $\left(A_{0}+A_{3}\right) / \sqrt{2}=0$ is taken as the gauge fixing condition, and have shown that residual gauge degrees of freedom are indispensable to implementing the Mandelstam-Leibbrandt (ML) prescription $[4,5]$ to the spurious singularities of the gauge field propagator. They also show that with the residual gauge degrees of freedom, equivalence with Feynman methods holds up to the one loop order. One therefore expects that a consistent light-front spatial gauge formulation, where $x_{l}^{+}$is chosen as the evolution parameter and $A_{-}^{(l)}=\left(A_{0}-A_{3}\right) / \sqrt{2}=0$ is taken as the gauge fixing condition, can also be constructed by introducing the residual gauge degrees of freedom.

However, one notices that because the residual gauge functions in the light-cone spatial gauge formulation depend only on $x_{l}^{+}, x^{1}$ and $x^{2}$, one cannot obtain dynamical operators by integrating 
densities over the three dimensional hyperplane $x_{l}^{+}=$constant. Therefore, it is essential to extend the conventional way of constructing the Hamiltonian to include the residual gauge fields. McCartor and Robertson [6] obtained the Hamiltonian by integrating the divergence equation of the energy-momentum tensor over a suitable closed surface and found that the translational generator $P_{+}$consists of physical degrees of freedom integrated over the hyperplane $x_{l}^{+}=$constant and residual degrees of freedom integrated over the hyperplane $x_{l}^{-}=\left(x^{0}-x^{3}\right) / \sqrt{2}=$ constant. However they have not succeeded in introducing the residual gauge fields' interactions consistently.

Because axial gauges of the type $n \cdot A=0$ could be viewed as continuous deformations of the light-cone gauge, Lazzizzera [7] has extended the canonical light-cone gauge formulation outside the light-cone gauge in ordinary coordinates and implemented the ML prescription with the help of the residual gauge fields. He has also noticed that in space-like axial gauge formulations, infrared divergences resulting from physical degrees of freedom are cancelled by those from the residual gauge degrees of freedom.

The aim of this contribution is to find ways to introduce consistently the residual gauge fields into the light-front interacting gauge theories. For simplicity we confine ourselves to QED and investigate light-front limits of canonical formulations of the axial gauge fields specified by $n \cdot A=0$ in $\tau \sigma$-coordinates where $x^{\mu}=\left(x^{\tau}, x^{\sigma}, x^{1}, x^{2}\right)$ with

$x^{\tau}=x^{0} \sin \phi+x^{3} \cos \phi, x^{\sigma}=x^{0} \cos \phi-x^{3} \sin \phi .(1)$

A similar framework was used by others to analyze two-dimensional models [8] and by $\mathrm{Ji}$ and Mitchell [9] to construct the Poincaré algebra interpolating between ordinary time and light-cone time quantizations. In this framework we have two independent free parameters at hand, so that we can quantize axial gauge fields on surfaces other than $x^{0}=$ constant. In fact, taking $x^{\sigma}$, in the region $0 \leq \phi<\pi / 4$, and $x^{\tau}$, in the region $\pi / 4<\phi \leq \pi / 2$ respectively as the evolution parameter enables us to construct, in quite the same manner as in ordinary coordinates, canonical formulations, in which the ML prescription is implemented and in which the infrared divergences are regularized. Especially, we find that in case that $n^{2}=0$, an explicit quantization surface dependence does not appear at all. Consequently, we obtain that the light-front temporal gauge limit $\phi \rightarrow \pi / 4-0$ of the $n^{2}=0$ case agrees with the light-front spatial gauge limit $\phi \rightarrow \pi / 4+0$ and that in both limits the free Hamiltonian consists of physical degrees of freedom integrated over the hyperplane $x_{l}^{+}=\left(x^{0}+x^{3}\right) / \sqrt{2}=$ constant and residual degrees of freedom integrated over the hyperplane $x_{l}^{-}=\left(x^{0}-x^{3}\right) / \sqrt{2}=$ constant. Furthermore, we find that in both limits, propagators of fermion and gauge fields have no contact terms, which reflects the fact that in genuine light-front perturbation calculations contact terms of fermion propagators are cancelled by contact terms resulting from the noncovariant interaction term and that those of gauge field propagators are cancelled by terms included in the Coulomb interaction term.

Before entering into details we denote our conventions. The constant vector $n^{\mu}$ is specified in the ordinary coordinates to be $\left(n^{0}, n^{3}, n^{1}, n^{2}\right)=$ $(\cos \theta,-\sin \theta, 0,0)$, which yields $n^{2}=\cos 2 \theta$. The temporal, light-cone and axial gauges are realized respectively when $\theta=0, \pi / 4$ and $\pi / 2$. In the $\tau \sigma$-coordinates the $n^{\mu}$ is expressed due to (1) as $\left(n^{\tau}, n^{\sigma}, n^{1}, n^{2}\right)=(\sin (\phi-\theta), \cos (\phi-\theta), 0,0)$ and lower indices are given by the help of the metric tensor

$g_{\sigma \sigma}=-g_{\tau \tau}=\cos 2 \phi, g_{\sigma \tau}=g_{\tau \sigma}=\sin 2 \phi$.

To denote the component $n \cdot A$ simply as $A_{-}$, we define +--coordinates by $x^{\mu}=\left(x^{+}, x^{-}, x^{1}, x^{2}\right)$, where

$x^{+}=x^{0} \sin \theta+x^{3} \cos \theta, x^{-}=x^{0} \cos \theta-x^{3} \sin \theta$. (3)

$x^{+}$and $x^{-}$are described in terms of $x^{\tau}$ and $x^{\sigma}$ as

$x^{+}=\cos (\phi-\theta) x^{\tau}-\sin (\phi-\theta) x^{\sigma}$

$x^{-}=\sin (\phi-\theta) x^{\tau}+\cos (\phi-\theta) x^{\sigma}$.

Latin indices $i, j$ will label the 1,2 component of a given four-vector or tensor and Latin indices $r, s$ will label the $\sigma, 1,2$ components a given fourvector or tensor. We furthermore use the following conventions; 
$\boldsymbol{x}^{\sigma}=\left(x^{\sigma}, x^{1}, x^{2}\right), d^{3} \boldsymbol{x}^{\sigma}=d x^{1} d x^{2} d x^{\sigma}$,

$\boldsymbol{k}_{\sigma}=\left(k_{\sigma}, k_{1}, k_{2}\right), d^{3} \boldsymbol{k}_{\sigma}=d k_{1} d k_{2} d k_{\sigma}$,

$\boldsymbol{k}_{+}=\left(k_{+}, k_{1}, k_{2}\right), d^{3} \boldsymbol{k}_{+}=d k_{1} d k_{2} d k_{+}$

\section{Canonical formulation of $n \cdot A=0$ gauge QED in $\tau \sigma$ - coordinates}

The $n \cdot A=0$ gauge formulation of QED is defined by the Lagrangian

$L=-\frac{1}{4} F_{\mu \nu} F^{\mu \nu}-B(n \cdot A)+\bar{\psi}\left(i \gamma^{\mu} D_{\mu}-m\right) \psi$

where $D_{\mu}=\partial_{\mu}+i e A_{\mu}$ and $B$ is the NakanishiLautrup field in the axial gauge formulations. Field equations and the gauge fixing condition are obtained to be

$\partial_{\mu} F^{\mu \nu}=n^{\nu} B+J^{\nu}$

$\left(i \gamma^{\mu} D_{\mu}-m\right) \psi=0$

$A_{-}=\cos (\theta-\phi) A_{\sigma}-\sin (\theta-\phi) A_{\tau}=0$.

The field equation of $B$,

$n \cdot \partial B=\partial_{-} B=0$,

is obtained by operating on (6) with $\partial_{\nu}$.

From (8) we see that as long as $\theta \neq \phi$, we can choose either $A_{\sigma}$ or $A_{\tau}$ as one of independent canonical variables. Actually $A_{\sigma}$ becomes the time component, and hence the dependent one, in the region $0 \leq \phi<\pi / 4$ due to the fact that $x^{\sigma}$ is chosen as the evolution parameter in that region, while $A_{\tau}$ becomes the dependent one in the region $\pi / 4<\phi \leq \pi / 2$. If we take these facts into account, we can construct the canonical formulation in both regions in quite the same manner as in the ordinary coordinates; therefore in what follows we only present the latter formulation.

In the region $\pi / 4<\phi \leq \pi / 2$ we can choose $x^{\tau}$ as the evolution parameter. Canonical conjugate momenta turn out to be

$$
\begin{aligned}
& \pi^{\tau}=0, \quad \pi^{\sigma}=F_{\tau \sigma}, \quad \pi^{i}=F_{i}^{\tau}, \\
& \pi_{B}=0, \quad \pi_{\psi}=i \bar{\psi} \gamma^{\tau}, \quad \pi_{\psi^{*}}=0
\end{aligned}
$$

and as long as $\theta \neq \phi$, we can choose $A_{s}, \pi^{s}$ ( $s=$ $1,2, \sigma)$ and $\psi, \psi^{*}$ as independent canonical variables and eliminate the dependent ones by

$A_{\tau}=\cot (\theta-\phi) A_{\sigma}, B=\left(\partial_{s} \pi^{s}-J^{\tau}\right) / n^{\tau}$.
Consequently, equal $x^{\tau}$-time canonical quantization conditions can be imposed on the independent canonical variables; the nonvanishing commutators are

$$
\begin{gathered}
{\left[A_{r}(x), \pi^{s}(y]=i \delta_{r s} \delta^{(3)}\left(\boldsymbol{x}^{\sigma}-\boldsymbol{y}^{\sigma}\right),\right.} \\
\left\{\psi(x), \psi^{*}(y)\right\}=\frac{\gamma^{0} \gamma_{\tau}}{-\cos 2 \phi} \delta^{(3)}\left(\boldsymbol{x}^{\sigma}-\boldsymbol{y}^{\sigma}\right) .
\end{gathered}
$$

The Hamiltonian, $H=H_{0}+H_{I}$, is given in terms of the independent canonical variables by

$$
\begin{gathered}
H_{0}=\frac{\left(\pi^{\sigma}\right)^{2}}{2}+\frac{\left(\pi^{i}-\sin 2 \phi F_{\sigma i}\right)^{2}}{-2 \cos 2 \phi}+\frac{-\cos 2 \phi}{2}\left(F_{\sigma i}\right)^{2} \\
+\frac{1}{2}\left(F_{12}\right)^{2}+\pi^{s} \partial_{s} A_{\tau}+\bar{\psi}\left(m-i \gamma^{s} \partial_{s}\right) \psi \\
H_{I}=e A_{\mu} \bar{\psi} \gamma^{\mu} \psi .
\end{gathered}
$$

To restore the Gauss law in the physical subspace, we Fourier-expand $B$, which satisfies (9) and hence depends only on $x^{+}=\cos (\theta-\phi) x^{\tau}+$ $\sin (\theta-\phi) x^{\sigma}, x^{1}$ and $x^{2}$, in the form

$$
\begin{gathered}
B(x)=\frac{1}{\sqrt{(2 \pi)^{3}}} \int \frac{d^{3} \boldsymbol{k}_{+}}{\sqrt{k_{+}}} \theta\left(k_{+}\right)\left(k_{\perp}^{2}+n^{2} k_{+}^{2}\right) \\
\times\left\{B\left(\boldsymbol{k}_{+}\right) e^{-i k \cdot x}+B^{*}\left(\boldsymbol{k}_{+}\right) e^{i k \cdot x}\right\}
\end{gathered}
$$

where $k \cdot x=k_{+} x^{+}+k_{i} x^{i}=k_{\tau} x^{\tau}+k_{\sigma} x^{\sigma}+k_{i} x^{i}$ with

$k_{\tau}=k_{+} \cos (\theta-\phi), \quad k_{\sigma}=k_{+} \sin (\theta-\phi)$,

and define the physical subspace $\mathcal{V}_{\text {phys }}$ by

$\mathcal{V}_{\text {phys }}=\{\mid$ phys $\rangle\left|B\left(\boldsymbol{k}_{+}\right)\right|$phys $\left.\rangle=0\right\}$.

The $S$-operator is given by

$$
\begin{aligned}
S= & \sum_{n=0}^{\infty} \frac{(-i)^{n}}{n !} \int d^{4} x^{(1)} \cdots \int d^{4} x^{(n)} \\
& \times \mathrm{T}\left[H_{I}\left(x^{(1)}\right) \cdots H_{I}\left(x^{(n)}\right)\right] .
\end{aligned}
$$

In what follows, to avoid inessential complications, we use the same notations for the variables in the interaction picture as for those in the Heisenberg picture.

\section{Quantization of the free gauge field in the $\tau \sigma$-coordinates}

We can canonically quantize the free gauge field in the same manner as we did in [10]. We begin by solving the free gauge field equations

$\partial_{\mu} F^{\mu \nu}=n^{\nu} B$ 
under the constraints

$n \cdot \partial B=\partial_{-} B=0, \quad n \cdot A=A_{-}=0$.

On multiplying (19) with $n_{\nu}$ we obtain

$-_{-}\left(\partial^{\mu} A_{\mu}\right)=n^{2} B$

due to the gauge fixing condition $A_{-}=0$. Then multiplying (21) with $\partial_{-}$provides us with

$\left(\partial_{-}\right)^{2}\left(\partial^{\mu} A_{\mu}\right)=0$

because $\partial_{-} B=0$. It follows from (22) and $\partial_{-} B=0$ that $A^{\nu}$ is required to satisfy

$\square\left(\partial_{-}\right)^{2} A^{\nu}=0$.

We see from (23) that $A^{\nu}$ consists of a free massless part and a linear function of $x^{-}$. It turns out that the free massless part, which we denote as $u^{\nu}$, satisfies

$n \cdot u=0, \quad \partial^{\mu} u_{\mu}=0, \quad \square u=0$

and that $x^{-}$has to be expressed in terms of $x^{\tau}$ and $x^{+}$as

$x^{-}=-\frac{x^{\tau}}{\sin (\theta-\phi)}+\frac{\cos (\theta-\phi)}{\sin (\theta-\phi)} x^{+}$

for $A^{\nu}$ to satisfy the equal $x^{\tau}$-time quantization conditions. Consequently we obtain

$A_{\mu}(x)=u_{\mu}(x)-\frac{n_{\mu}}{\nabla^{2}} B(x)+\partial_{\mu} \Gamma(x)$

where

$$
\begin{aligned}
\nabla^{2} & =\partial_{1}{ }^{2}+\partial_{2}{ }^{2}+\frac{n^{2} \partial_{\sigma}{ }^{2}}{\sin ^{2}(\theta-\phi)} \\
\Gamma & =\frac{-1}{\nabla^{2}}\left(C+\frac{n^{2} x^{\tau}}{\sin (\theta-\phi)} B-\frac{n^{2} n_{\sigma}}{\sin ^{2}(\theta-\phi) \nabla^{2}} \partial_{\sigma} B\right)
\end{aligned}
$$

From (24) we see that two degrees of freedom are carried by $u_{\mu}$, so that the remaining degrees of freedom are carried by a pair of residual gauge fields $B$ and $C$, which depend on $x^{+}, x^{1}$ and $x^{2}$. As a matter of fact, the equal $x^{\tau}$-time quantization conditions require that $u_{\mu}, B$ and $C$ are fundamental fields satisfing the following commutation relations:

$$
\begin{gathered}
{\left[u_{\mu}(x), u_{\nu}(y)\right]=i\left(-g_{\mu \nu}+\frac{n_{\mu} \partial_{\nu}+n_{\nu} \partial_{\mu}}{\partial_{-}}\right.} \\
\left.-n^{2} \frac{\partial_{\mu} \partial_{\nu}}{\partial_{-}{ }^{2}}\right) D(x-y),
\end{gathered}
$$

$$
[B(x), C(y)]=-i \nabla^{2} \delta^{(3)}\left(\boldsymbol{x}^{+}-\boldsymbol{y}^{+}\right)
$$

where $D(x)$ is the commutator function of the free massless field and $\boldsymbol{x}^{+}=\left(x^{+}, x^{1}, x^{2}\right)$. All other commutators among $u_{\mu}, B$ and $C$ are zero. Consequently $A_{\mu}$ satisfies the following 4-dimensional commutation relations

$$
\begin{gathered}
{\left[A_{\mu}(x), A_{\nu}(y)\right]=i\left\{-g_{\mu \nu} D(x-y)+\left(n_{\mu} \partial_{\nu}\right.\right.} \\
\left.\left.+n_{\nu} \partial_{\mu}\right) \partial_{-} E(x-y)-n^{2} \partial_{\mu} \partial_{\nu} E(x-y)\right\}
\end{gathered}
$$

where

$$
\begin{gathered}
E(x)=\frac{1}{\partial_{-}{ }^{2}} D(x)-\frac{1}{\nabla^{2}}\left(D_{s}(x)\right. \\
\left.+\frac{2 n_{\sigma}}{\sin ^{2}(\theta-\phi) \nabla^{2}} \partial_{\sigma} \partial_{-} D_{s}(x)\right)
\end{gathered}
$$

with

$D_{s}(x)=-\frac{x^{\tau}}{\sin (\theta-\phi)} \delta^{(3)}\left(\boldsymbol{x}^{+}\right)$

Other properties of $A_{\mu}$ are enumerated as follows:

(1) As a consequence of the fact that dipole ghost fields cannot be manifest Lorentz scalars, explicit quantization surface dependences appear in the second and third terms of the $\Gamma$, except for the case of $n^{2}=0$.

(2) The factor $x^{\tau} / \sin (\theta-\phi)$ of the second term of $\Gamma$ is not well-defined in the limit $\phi \rightarrow \theta$, which reflects the fact that we cannot construct the canonical formulation in the same manner in that limit. Actually $A_{\sigma}$ vanishes in that limit and as a result $\pi^{\sigma}=F_{\tau \sigma}$ becomes a constraint. Whereas in the region $0 \leq \phi<\pi / 4$ the limit $\phi \rightarrow \theta$ is well-defined so that we can construct the temporal gauge formulation in the $\tau \sigma$-coordinates.

(3) When $\theta$ tends to $\phi$ in the region $\pi / 4<\phi \leq \pi / 2$, the gauge $A_{-}=0$ becomes a pure space-like axial gauge, so that we encounter the same difficulty as that in the ordinary coordinates. We notice however, that the quantization surface dependences disappear when we calculate the $x^{\tau}$ time ordered propagator in momentum space, so that we can obtain well-defined limits in momentum space. We show this below.

(4) Translational generators for the free gauge field are described as follows:

$$
\begin{aligned}
P_{\tau} & =\int d^{3} \boldsymbol{x}^{\sigma}\left[\theta_{\tau}{ }^{\sigma}-\frac{1}{2} B \frac{n^{2}}{\nabla^{2}} B-B \frac{n^{\sigma}}{\nabla^{2}} \partial_{\sigma} C\right], \\
P_{s} & =\int d^{3} \boldsymbol{x}^{\sigma}\left[\theta_{s}{ }^{\sigma}+B \frac{n^{\tau}}{\nabla^{2}} \partial_{s} C\right]
\end{aligned}
$$


where $s=\sigma, 1,2$ and $\theta_{\tau}{ }^{\sigma}$ and $\theta_{s}{ }^{\sigma}$ are the canonical energy-momentum tensor composed only of the physical $u^{\mu}$. Due to the second term of $P_{\tau}$, we obtain the Heisenberg equation $\left[\Gamma(x), P_{\tau}\right]=$ $i \partial_{\tau} \Gamma(x)$ in spite of the fact that $\Gamma$ has the explicit $x^{\tau}$ dependence.

(5) The operator $\nabla^{2}$ becomes hyperbolic when $n^{2}<0$. We regularize singularities of its inverse by the Cauchy principal value prescription.

(6) The inverse of $\nabla^{2}$ also appears in the physical part as we see from the fact that the operator $\frac{1}{\partial_{-}}$ in (29) and (32) is rewritten for $D(x)$ as

$$
\begin{gathered}
\frac{1}{\partial_{-}}=\frac{\cos (\theta+\phi) \partial_{\sigma}-\sin (\theta-\phi) \partial^{\tau}}{\sin ^{2}(\theta-\phi) \nabla^{2}} \\
=\frac{\cos (\theta+\phi) \partial_{\sigma}-\sin (\theta-\phi) \partial^{\tau}}{\partial_{\perp}^{2} \sin ^{2}(\theta-\phi)+n^{2} \partial_{\sigma}{ }^{2}} .
\end{gathered}
$$

(7) Therefore when $n^{2}<0$, infrared divergences necessarily appear in the physical part irrespective of the quantization surface. These divergences are cancelled by corresponding ones from the residual gauge field's part.

Properties in the $n^{2}=0$ case are given separately in section 5 . We close this section by calculating the $x^{\tau}$-time ordered propagator of the gauge field. The constituent fields are expressed in terms of creation and annihilation operators. From (29) we see that $u_{\mu}$ can be written as

$$
\begin{gathered}
u_{\mu}(x)=\frac{1}{\sqrt{2(2 \pi)^{3}}} \int \frac{d^{3} \boldsymbol{q}_{\sigma}}{\sqrt{q^{\tau}}} \sum_{\lambda=1}^{2} \epsilon_{\mu}^{(\lambda)}(q) \\
\times\left\{a_{\lambda}\left(\boldsymbol{q}_{\sigma}\right) e^{-i q \cdot x}+a_{\lambda}^{*}\left(\boldsymbol{q}_{\sigma}\right) e^{i q \cdot x}\right\}
\end{gathered}
$$

where $q^{\tau}$ is the on mass-shell energy given by

$q^{\tau}=\sqrt{q_{\sigma}^{2}-\cos 2 \phi q_{\perp}^{2}}$

and $q^{\sigma}$ and $q_{\tau}$ are expressed in terms of $q_{\sigma}$ and $q^{\tau}$ respectively as

$q^{\sigma}=\frac{q_{\sigma}-\sin 2 \phi q^{\tau}}{\cos 2 \phi}, q_{\tau}=\frac{\sin 2 \phi q_{\sigma}-q^{\tau}}{\cos 2 \phi}$.

The polarization vector $\epsilon_{\mu}^{(\lambda)}$ satisfies

$$
\begin{gathered}
q^{\mu} \epsilon_{\mu}^{(\lambda)}(q)=0, \quad n^{\mu} \epsilon_{\mu}^{(\lambda)}(q)=0, \quad(\lambda=1,2) \\
\sum_{\lambda=1}^{2} \epsilon_{\mu}^{(\lambda)}(q) \epsilon_{\nu}^{(\lambda)}(q) \\
=-g_{\mu \nu}+\frac{n_{\mu} q_{\nu}+n_{\nu} q_{\mu}}{q_{-}}-n^{2} \frac{q_{\mu} q_{\nu}}{q_{-}{ }^{2}}
\end{gathered}
$$

where $q_{-}=\cos (\theta-\phi) q_{\sigma}-\sin (\theta-\phi) q_{\tau} . B$ and $C$ are expressed in terms of zero-norm creation and annihilation operators as follows

$$
\begin{gathered}
B(x)=\frac{1}{\sqrt{(2 \pi)^{3}}} \int \frac{d^{3} \boldsymbol{k}_{+}}{\sqrt{k_{+}}} \theta\left(k_{+}\right)\left(k_{\perp}^{2}+n^{2} k_{+}^{2}\right) \\
\times\left\{B\left(\boldsymbol{k}_{+}\right) e^{-i k \cdot x}+B^{*}\left(\boldsymbol{k}_{+}\right) e^{i k \cdot x}\right\}, \\
C(x)=\frac{i}{\sqrt{(2 \pi)^{3}}} \int d^{3} \boldsymbol{k}_{+} \sqrt{k_{+}} \theta\left(k_{+}\right) \\
\times\left\{C\left(\boldsymbol{k}_{+}\right) e^{-i k \cdot x}-C^{*}\left(\boldsymbol{k}_{+}\right) e^{i k \cdot x}\right\},
\end{gathered}
$$

where

$$
\begin{aligned}
& {\left[B\left(\boldsymbol{k}_{+}\right), C^{*}\left(\boldsymbol{q}_{+}\right)\right]=-\delta^{(3)}\left(\boldsymbol{k}_{+}-\boldsymbol{q}_{+}\right)} \\
& {\left[C\left(\boldsymbol{k}_{+}\right), B^{*}\left(\boldsymbol{q}_{+}\right)\right]=-\delta^{(3)}\left(\boldsymbol{k}_{+}-\boldsymbol{q}_{+}\right) .}
\end{aligned}
$$

Consequently we can calculate the $x^{\tau}$-time orderd propagator

$$
\left\langle 0\left|T\left(A_{\mu}(x) A_{\nu}(y)\right)\right| 0\right\rangle=\int \frac{d^{4} q}{(2 \pi)^{4}} T_{\mu \nu}(q) e^{-i q \cdot(x-y)} .
$$

We must keep $\theta<\phi$ in order for all the terms containing the inverse of $\nabla^{2}$ to cancel. Thus, specifying $\theta$ and $\phi$ as required, we obtain

$$
T_{\mu \nu}(q)=\frac{i}{q^{2}+i \varepsilon}\left(-g_{\mu \nu}+\frac{n_{\mu} q_{\nu}+n_{\nu} q \mu}{\left[q_{-}\right]}-\frac{n^{2} q_{\mu} q_{\nu}}{\left[q_{-}\right]^{2}}\right)
$$

where

$\left[q_{-}\right]=q_{-}+i \varepsilon \operatorname{sgn}\left(q_{+}\right)$.

It is remarkable that the ML prescription has been realized in the manner which does not depend on the quantization surface. Consequently we can take the limit $\phi \rightarrow \theta$ of the propagator and define it to be that of the gauge field in the pure space-like axial gauge formulation in the $\tau \sigma$ - coordinates. We can similarly define that of the pure space-like axial gauge field in the ordinary coordinates. We keep the relation $\theta<\phi$ by first fixing the value of $\phi$ to be $\pi / 2$ and then by taking the limit $\theta \rightarrow \pi / 2$ and obtain

$i \frac{-g_{\mu \nu}+\frac{n_{\mu} q_{\nu}+n_{\nu} q \mu}{\left[q_{3}\right]}+\frac{q_{\mu} q_{\nu}}{\left[q_{3}\right]^{2}}}{q^{2}+i \varepsilon}$

where $\left[q_{3}\right]=-q_{3}+i \varepsilon \operatorname{sgn}\left(q_{0}\right)$. 


\section{Quantization of the free Dirac field and the light-front limit}

We employ the following representation of the Dirac's matrices:

$$
\begin{aligned}
& \gamma^{0}=i\left(\begin{array}{cc}
0 & -I \\
I & 0
\end{array}\right), \quad \gamma^{3}=i\left(\begin{array}{cc}
0 & I \\
I & 0
\end{array}\right), \\
& \gamma^{1}=i\left(\begin{array}{cc}
-\sigma_{1} & 0 \\
0 & \sigma_{1}
\end{array}\right), \gamma^{2}=i\left(\begin{array}{cc}
-\sigma_{2} & 0 \\
0 & \sigma_{2}
\end{array}\right)
\end{aligned}
$$

where $I$ is $2 \times 2$ unit matrix. Matrices $\gamma^{\tau}$ and $\gamma^{\sigma}$ are defined by

$\gamma^{\tau}=\gamma^{0} \sin \phi+\gamma^{3} \cos \phi, \gamma^{\sigma}=\gamma^{0} \cos \phi-\gamma^{3} \sin \phi(48)$

and then $\gamma \cdot p-m$ is expressed as

$\gamma \cdot p-m=i\left(\begin{array}{cc}-\left(m+i \sigma_{i} p_{i}\right) & -i \frac{p^{\tau}+p_{\sigma}}{\sqrt{1+\sin 2 \phi}} \\ i \frac{p^{\tau}-p_{\sigma}}{\sqrt{1-\sin 2 \phi}} & -\left(m-i \sigma_{i} p_{i}\right)\end{array}\right)$

so that the plane wave solutions of the free Dirac equation are obtained in the same manner as in the ordinary coordinates. It turns out that the positive energy solution, $u^{(r)}\left(\boldsymbol{p}_{\sigma}\right)(r=1,2)$, and the negative energy solutions, $v^{(r)}\left(\boldsymbol{p}_{\sigma}\right)(r=1,2)$, are given by

$$
\begin{aligned}
& u^{(r)}\left(\boldsymbol{p}_{\sigma}\right)=N_{u}\left(\begin{array}{c}
\chi^{(r)} \\
i \frac{\sqrt{1+s}}{p^{\tau}+p_{\sigma}}\left(m+i \sigma_{i} p_{i}\right) \chi^{(r)}
\end{array}\right), \\
& v^{(r)}\left(\boldsymbol{p}_{\sigma}\right)=N_{v}\left(\begin{array}{c}
i \frac{\sqrt{1-s}}{p^{\tau}-p_{\sigma}}\left(m+i \sigma_{i} p_{i}\right) \chi^{(r)} \\
\chi^{(r)}
\end{array}\right)
\end{aligned}
$$

where $p^{\tau}=\sqrt{p_{\sigma}^{2}-\cos 2 \phi\left(p_{\perp}^{2}+m^{2}\right)}, \chi^{(r)}$ is the two-component spinor, $s=\sin 2 \phi$ and $N_{u}$ and $N_{v}$ are normalization factors given by

$N_{u}=\sqrt{\frac{p^{\tau}+p_{\sigma}}{2 m \sqrt{1+s}}}, \quad N_{v}=\sqrt{\frac{p^{\tau}-p_{\sigma}}{2 m \sqrt{1-s}}}$.

We see that when $p_{\sigma}<0$, the lower twocomponent spinor is not well-defined in the limit $\phi \rightarrow \pi / 4$. This reflects the fact that the lower two-component spinor cannot be an independent canonical variable in the light-front formulation.

Nevertheless, the $x^{\tau}$-time ordered propagator of the free Dirac field $\psi$ is well-defined in the limit. As a matter of fact, because the $\psi$ is described in terms of the particle annihilation operator $b_{(r)}\left(\boldsymbol{p}_{\sigma}\right)$ and the antipaticle creation operator

$$
\begin{aligned}
& d_{(r)}^{*}\left(\boldsymbol{p}_{\sigma}\right) \text { as } \\
& \begin{aligned}
\psi=\int \frac{d^{3} \boldsymbol{p}_{\sigma}}{(2 \pi)^{\frac{3}{2}}} & \sqrt{\frac{m}{p^{\tau}}} \sum_{r=1}^{2}\left\{b_{(r)}\left(\boldsymbol{p}_{\sigma}\right) u^{(r)}\left(\boldsymbol{p}_{\sigma}\right) e^{-i p \cdot x}\right. \\
& \left.+d_{(r)}^{*}\left(\boldsymbol{p}_{\sigma}\right) v^{(r)}\left(\boldsymbol{p}_{\sigma}\right) e^{i p \cdot x}\right\}
\end{aligned}
\end{aligned}
$$

we obtain the $x^{\tau}$-time ordered propagator

$$
\langle 0| T(\psi(x) \bar{\psi} y))|0\rangle=\int \frac{d^{4} q}{(2 \pi)^{4}} S(q) e^{-i q \cdot(x-y)}
$$

where $S(q)$ consists of the particle and antiparticle contributions in the form

$$
\begin{array}{r}
S(q)=i \frac{\gamma^{\tau} p_{\tau}\left(\boldsymbol{q}_{\sigma}\right)+\gamma^{\sigma} q_{\sigma}+\gamma^{i} q_{i}+m}{2 p^{\tau}\left(\boldsymbol{q}_{\sigma}\right)\left(q_{\tau}-p_{\tau}\left(\boldsymbol{q}_{\sigma}\right)+i \varepsilon\right)} \\
+\quad i \frac{\gamma^{\tau} p_{\tau}\left(-\boldsymbol{q}_{\sigma}\right)-\gamma^{\sigma} q_{\sigma}-\gamma^{i} q_{i}-m}{2 p^{\tau}\left(\boldsymbol{q}_{\sigma}\right)\left(q_{\tau}+p_{\tau}\left(-\boldsymbol{q}_{\sigma}\right)-i \varepsilon\right)}
\end{array}
$$

with

$p_{\tau}\left(\boldsymbol{q}_{\sigma}\right)=\frac{p^{\tau}\left(\boldsymbol{q}_{\sigma}\right)-\sin 2 \phi q_{\sigma}}{-\cos 2 \phi}$.

Thus they combine to give the standard expression

$S(q)=i \frac{\gamma \cdot q+m}{q^{2}-m^{2}+i \varepsilon}$

from which follows the covariant part of the lightfront fermion propagator in the limit. Moreover, analizing the limit $\phi \rightarrow \pi / 4$ of (53), we see that contact terms have appeared in the limit but they have cancelled among themselves. In fact, when $q_{\sigma} \leq 0, p_{\tau}\left(\boldsymbol{q}_{\sigma}\right)$ diverges in the limit, as is seen from

$\lim _{\phi \rightarrow \frac{\pi}{4}} p_{\tau}\left(\boldsymbol{q}_{\sigma}\right)= \begin{cases}\frac{q_{\perp}^{2}+m^{2}}{2 q_{\sigma}} & \left(q_{\sigma}>0\right) \\ +\infty & \left(q_{\sigma} \leq 0\right)\end{cases}$

the divergent $p_{\tau}\left(\boldsymbol{q}_{\sigma}\right)$ gives rise to a finite contact term

$$
\lim _{\theta \rightarrow \frac{\pi}{4}} S(q)=i \frac{\gamma^{\tau} \frac{q_{\perp}^{2}+m^{2}}{2 q_{\sigma}}+\gamma^{\sigma} q_{\sigma}+\gamma^{i} q_{i}+m}{q^{2}-m^{2}+i \varepsilon}+\frac{i \gamma^{\tau}}{2 q_{\sigma}} .
$$

However, in the limit $q^{2}=2 q_{\tau} q_{\sigma}-q_{\perp}^{2}$, it cancels the one resulting from the first term, as is seen from

$\frac{\gamma^{\tau}}{2 q_{\sigma}}\left(1+\frac{q_{\perp}^{2}+m^{2}}{q^{2}-m^{2}+i \varepsilon}\right)=\frac{\gamma^{\tau} q_{\tau}}{q^{2}-m^{2}+i \varepsilon}$.

We can conclude from (55) that we can obtain the covariant fermion propagator irrespective of the quantization surface as long as the lower twocomponent spinor is the independent canonical variable. 


\section{Light-front formulation of light-cone gauge QED}

As has been pointed out in section 4 , in case of $n^{2}=0$, namely the light-cone gauge, the manifest quantization surface dependences disappear from the free gauge field, as is seen from

$A_{\mu}=u_{\mu}-\frac{n_{\mu}}{\nabla_{\perp}^{2}} B-\frac{\partial_{\mu}}{\nabla_{\perp}^{2}} C$

where $\nabla_{\perp}{ }^{2}=\partial_{1}{ }^{2}+\partial_{2}{ }^{2}$. Accordingly, the second term of $P_{\tau}$ in (34) also vanishes so that if we calculate quantities made of the residual gauge fields by using equal $x^{\tau}$-time commutation relations, then the quantities do not have $x^{\tau}$ dependences so that we can obtain the same results by using the translational generators with the integration variable $d x^{\sigma}$ changed into $d x^{+} / \sin \left(\frac{\pi}{4}-\phi\right)$. Thus, in that sense, the translational generators can be identified with

$$
\begin{aligned}
P_{\tau} & =\left[\int d^{3} \boldsymbol{x}^{\sigma} \theta_{\tau}{ }^{\sigma}-\int d^{3} \boldsymbol{x}^{+} B \frac{\cos \left(\frac{\pi}{4}-\phi\right)}{\nabla_{\perp}{ }^{2}} \partial_{+} C\right] \\
P_{s} & =\left[\int d^{3} \boldsymbol{x}^{\sigma} \theta_{s}{ }^{\sigma}-\int d^{3} \boldsymbol{x}^{+} B \frac{1}{\nabla_{\perp}{ }^{2}} \partial_{s} C\right]
\end{aligned}
$$

where the second term of $P_{\tau}$ has been obtained by using $\partial_{\sigma}=\sin \left(\frac{\pi}{4}-\phi\right) \partial_{+}$. Now we can obtain the light-front limit $\phi \rightarrow \pi / 4$

$$
\begin{aligned}
& P_{\tau}=\left[\int d^{3} \boldsymbol{x}^{\sigma} \theta_{\tau}{ }^{\sigma}-\int d^{3} \boldsymbol{x}^{+} B \frac{1}{\nabla_{\perp}^{2}} \partial_{+} C\right], \\
& P_{s}=\left[\int d^{3} \boldsymbol{x}^{\sigma} \theta_{s}{ }^{\sigma}-\int d^{3} \boldsymbol{x}^{+} B \frac{1}{\nabla_{\perp}^{2}} \partial_{s} C\right] .
\end{aligned}
$$

These are equations given previously by McCartor and Robertson [6]. Now that we have the free Hamiltonian, we can construct the light-front perturbation theory of light-cone gauge QED by employing the light-front limit of the lightcone gauge interaction Hamiltonian as that of the light-front theory. By construction, the free gauge field possesses $x^{-}$independent residual gauge fields regularizing the infrared divergences so that we obtain a well-defined gauge field propagator [10]. Note that the regularizations are done most effectively by carrying out $x^{-}$-integrations after calculating the propagators. Then we obtain $\frac{i}{q^{2}+i \varepsilon}\left(-g_{\mu \nu}+\frac{n_{\mu} q_{\nu}+n_{\nu} q \mu}{\left[q_{-}\right]}\right)$as the gauge field propagator and $i \frac{\gamma \cdot q+m}{q^{2}-m^{2}+i \varepsilon}$ as the fermion propagator.
We end this contribution by making remarks. In our formulation the light-front temporal gauge limit agrees with the light-front spatial gauge limit, because the free light-cone gauge field given in (60) is identical with that in the formulation where the $x^{\sigma}$ is chosen as the evolution parameter. From the fact that light-cone gauge QED gives rise to the covariant interaction term as well as the propagators without the contact terms in the light-front limit, we see that in the genuine light-front perturbation calculations the contact terms resulting from the fermion propagators are cancelled by the terms provided by the noncovariant interaction term

$e^{2} \bar{\psi} \gamma^{\mu} A_{\mu} \frac{\gamma^{+}}{2 i \partial_{-}} \gamma^{\nu} A_{\nu} \psi$

And the contact terms resulting from the gauge field propagators are cancelled by the terms provided by the Coulomb interaction term.

\section{REFERENCES}

1. N. Nakanishi, Phys. Lett. 131B (1983) 381.

2. A. Bassetto, M. Dalbosco, I. Lazzizzera and R. Soldati, Phys. Rev. D31 (1985) 2012.

3. M. Morara and R. Soldati, Phys. Rev. D58 (1998) 105011.

4. S. Mandelstam, Nucl. Phys. B213 (1983) 149.

5. G. Leibbrandt, Phys. Rev. D29 (1984) 1699.

6. G. McCartor and D. G. Robertson, Z. Phys. C62 (1994) 349; C68 (1995) 345.

7. I. Lazzizzera, Phys. Lett. B210 (1988) 188; Nuovo Cim. 102A (1989) 1385.

8. K. Hornbostel, Phys. Rev. D45 (1992) 3781; E. Prokhvatilov and V. Franke, Yad. Fiz. 49 (1989) 1109; F. Lenz, M. Thies, S. Levit and D. Yazaki, Ann. of Phys. 208 (1991) 1.

9. C.-R. Ji and C. Mitchell, Phys. Rev. D64 (2001) 085013.

10. Y. Nakawaki and G. McCartor, Prog. Theor. Phys. 102 (1999) 149. 